\title{
La calidad de vida en pacientes con esquizofrenia y sus familias. Análisis bioético, conceptual y psicopatológico.
}

\section{The quality of life in patients with schizophrenia and their} families. Bioethical, conceptual and psychopathological analysis.

\section{Sergio Ramos Pozón}

Facultad de Filosofía. Universidad de Barcelona.

\section{Resumen}

En este artículo queremos analizar la calidad de vida (CV) en los pacientes con esquizofrenia. Para ello, en primer lugar exponemos un marco ético que justifique por qué es necesaria la introducción de la CV en la praxis médica. En segundo lugar, intentamos clarificar qué se entiende por $C V$ y qué aspectos son los que hay que valorar. Por último, exponemos de qué manera la enfermedad mental y las relaciones interpersonales pueden incidir en la $\mathrm{CV}$ de los pacientes con enfermedades mentales graves.

Palabras clave. Bioética. Calidad de vida. Psicopatología. Salud mental. Esquizofrenia.

\section{Abstract}

In this paper we want to analyze the Quality of Life (QL) in patients with schizophrenia. First, to do this, we present an ethical framework to justify why the introduction of the $\mathrm{QL}$ in medical praxis is necessary. Second, we try to clarify what is meant by QL and what aspects are to be valued. Finally, we show how mental illness and interpersonal relationships can affect the QL of patients with severe mental illness.

Keywords. Bioethics. Quality of Life. Psychopathologie. Mental health. Schizophrenia 


\section{Aspectos éticos}

Cuando evaluamos la CV de las personas, la finalidad es detectar si hay satisfacción con los distintos ámbitos que caracterizan la vida cotidiana, para posteriormente integrar dicha información en los planes de cuidado. Ahora bien, al exponer los motivos por los que está o no satisfecha, se está indicando cuáles son sus intereses. Dworkin (Dworkin, 1993: 95) sostiene que existen intereses de experiencia e intereses críticos. Los primeros se refieren a aquellos intereses por los cuales las personas dirigen su vida y que hacen alusión a la obtención de placer o bienestar. Los segundos muestran un compromiso con la vida y con las otras personas, pues no se centran en la obtención de un interés placentero o de bienestar, sino que se busca analizar qué se debería desear para que una vida sea buena.

No se trata simplemente de basarnos en una ética de máximos que indique qué proyecto de vida buena queremos, sino que si la persona desea que sus decisiones sean respetadas, aquello que decida ha de ser acorde con aquellos criterios exigibles a todos los ciudadanos y que posibilitan la convivencia pacífica de los ciudadanos con diferentes morales, es decir, con una ética de mínimos. Y es que, como sostiene Camps (Camps, 2001: 56), no basta con elegir el bien, un bien estético, sino que hay que justificarlo, de modo que las elecciones sobre proyectos de vida no se refieren a un asunto individual, sino comunitario, pues es injusto elegir el bien a costa de los demás. Ahora bien, una vez que las personas deciden, y lo hacen responsablemente, es necesario que la sociedad pluralista como en la que vivimos reconozca el derecho a las personas de poder optar por un determinado modo de vida acorde con sus valores. Es preciso que la sociedad dé un valor fundamental a la tolerancia y al respeto por esas decisiones, que son proyectos de vida, y además que garantice que no ha de haber imposiciones.

En ocasiones, estas imposiciones pueden venir dadas por entender el valor de la vida

Ha de ser la persona quien elija y determine qué es lo valioso de la vida en relación con sus valores y preferencias, concibiéndola como un valor necesario, pues se trata de una vida de calidad entendida a su modo. de modo totalitario y/o religioso. Al analizar el valor de la vida podemos apreciar dos modos distintos de hacerlo. Dworkin (Dworkin, 1998: 97) opina que la vida puede ser considerada importante desde un punto de vista interno al sostener su sacralidad, es decir, que es intrínsecamente valiosa independientemente de lo que las personas quieran, deseen o necesiten. Esta es la postura religiosa que da un valor prioritario a la vida. Pero es posible también un enfoque liberal, que es el que defiende el autor, en el que las aportaciones humanas sean las que doten de significado y valor, de modo que "mi vida puede ser, para mí, más importante que ninguna otra cosa, pero de esto no se sigue que mi vida sea más importante intrínsecamente" (Dworkin, 1998: 110). Ha de ser la persona quien elija y determine qué es lo valioso de la vida en relación con sus valores y preferencias, concibiéndola como un valor necesario, pues se trata de una vida de calidad entendida a su modo. Al definir alguien qué entiende por valioso, está haciendo referencia a qué aspectos son beneficiosos y cuáles desea evitar porque los considera un mal. Llevar hasta las últimas consecuencias esta postura puede significar incluso elegir rechazar la vida (biológica) si ésta no es acorde con su proyecto de vida, es decir, con aquello que considera como una vida digna de ser vivida. En tal caso, es una obligación moral 
hacer todo lo posible para que la persona tenga un proceso de morir de manera digna, es decir, sin sufrimiento.

Al darle a la persona el protagonismo para poder definir qué entiende por $\mathrm{CV}$ se le está concibiendo como un ser dotado de autonomía que decide responsablemente sobre los acontecimientos de su vida. El reconocimiento y aceptación de esa decisión responsable es el respeto por la dignidad de la persona. Y si, efectivamente, queremos respetarle, hemos de asegurar de que no se le impondrá ninguna decisión, a no ser que haya motivos suficientes y justificados que supongan la necesidad de tomar decisiones en su lugar (1). Y es que "lo que sea bueno para cada cual es elegido libremente, nadie tiene derecho a decirlo ni imponerlo" (Camps, 2001: 38). Pero si no hay tales motivos suficientes y justificados que supongan la necesidad de tomar decisiones en su lugar, su decisión ha de ser respetada, porque está fundamentada en valores y preferencias que determinan cómo concibe una vida digna. Esa vida digna ha de estar compuesta por lo que Nussbaum (Nussbaum, 2007: 83) ha denominado capacidades humanas: "aquello que las personas son efectivamente capaces de hacer y ser, según una idea intuitiva de lo que es una vida acorde con la dignidad del ser humano". La lista se halla compuesta por las siguientes capacidades humanas: 1) vida; 2) salud física; 3) integridad física; 4) sentidos, imaginación y pensamiento; 5) emociones; 6) razón práctica; 7) afiliación; 8) otras especies; 9) juego; y 10) control sobre el propio entorno (Nussbaum, 2007: 88-89). Estas capacidades son las que dan sentido pleno a la manera de definir esa vida digna, de manera que hay que hacer todo lo posible para que se lleven a cabo. $Y$ es que "una sociedad que no las garantice a todos sus ciudadanos, en un nivel mínimo adecuado, no llega a ser una sociedad plenamente justa" (Nussbaum, 2007: 87).

Por tanto, lo que se está reivindicando es el derecho a una vida digna, de calidad, y no simplemente al derecho a la vida, que en ocasiones puede significar una vida biológica sin ningún tipo de sentido para la persona que la vive y/o sufre. El contenido de esa vida digna sólo puede concebirse desde la propia persona, en base a sus propios valores. $Y$ ese contenido se refiere a esas capacidades que menciona Nussbaum y

"El contenido de la vida digna sólo puede concebirse desde la propia persona, en base a sus propios valores. $Y$ ese contenido se refiere a las capacidades que menciona Nussbaum y que han de ser llevadas a cabo en los ámbitos micro, meso y macro.

han de ser llevadas a cabo en los ámbitos micro, meso y macro.

A continuación exponemos algunas nociones básicas sobre CV, con la finalidad de comprender, aunque sea mínimamente, qué entendemos por tal concepto.

\section{Concepto de calidad de vida}

El concepto de CV tiene un origen que se remonta a los griegos, aunque ellos utilizaban nociones como las de salud, bienestar, felicidad, etc. Pero es a partir de 1980 cuando propiamente se comienza a aplicar al ámbito de la salud, tomándose como eje la planificación centrada en la persona, a través de la evaluación de los resultados y la mejora de la calidad. En los años 80 la CV estaba orientada a promover la autodeterminación, inclusión, capacitación e igualdad de todas las personas. De esta manera se fue aplicando a los programas de servicios sociales centrados en la 
persona, a través de la mejora en la gestión de la calidad. Durante los años 90 se intentó conceptualizar y medir con mayor rigor, a fin de poder aplicarla a los planes de cuidados. Para ello se focalizó en temas como la igualdad, la inclusión, la capacitación, las oportunidades positivas de crecimiento, buscando basar este enfoque en evidencias (Schalock y Verdugo, 2009: 30-32).

Aunque llevamos más de 30 años utilizando el concepto de CV, carecemos de una definición precisa, y esto lleva a algunos autores a dudar sobre su aplicabilidad e incluso a admitir que es un constructo hipotético y no una entidad concreta (Hunt, 1997). Ciertamente, aunque hay acuerdo de que carecemos de una buena definición y de que las que hay son vagas e imprecisas, esto no significa que no tenga utilidad clínica para realizar buenos planes de salud y predecir la satisfacción de los pacientes.

En el intento de dar una definición operativa, la Organización Mundial de la Salud (1994) lo hizo del siguiente modo: "la percepción de los individuos de su posición en la vida en el contexto y sistema de valores en el cual ellos viven y en relación a sus metas, expectativas, normas y asuntos.

Es un concepto muy amplio que resulta afectado de modo complejo por la salud física de la persona, el estado psicológico, el nivel de independencia, las relaciones sociales, así como por los elementos esenciales de su entorno".

Al admitir el carácter multidimensional de la calidad de vida, se está asumiendo que entre sus componentes están las relaciones interpersonales, la inclusión social, el desarrollo personal, el bienestar físico, la autodeterminación, el bienestar material, el bienestar emocional y el disfrute de los derechos.
Pese a los intentos por definir la CV, sigue habiendo desacuerdo al respecto, razón por la cual la bibliografía ha optado por abandonar las pretensiones de realizar una definición precisa, para centrarse en el desarrollo de los ámbitos que abarca y en qué aspectos la promueven e incrementan. De este modo, se ha podido alcanzar un consenso sobre cuatro directrices que pueden resultar ser la base de su comprensión (Schalock y Verdugo, 2009: 32-40):

1. Asumir su carácter multidimensional y la necesidad de desarrollar indicadores referentes a las distintas áreas.

2. Evaluar tanto objetiva como subjetiva la vida de la persona.

3. Diferenciar los resultados a corto y a largo plazo.

4. Ceñirse a los predictores de los resultados de calidad.

Al admitir el carácter multidimensional, se está asumiendo que tiene componentes sobre relaciones interpersonales, inclusión social, desarrollo personal, bienestar físico, autodeterminación, bienestar material, bienestar emocional y derechos (2) (Schalock y Verdugo, 2002 y 2009). Aunque se puede discutir si estos aspectos son los que verdaderamente están vinculados a la $\mathrm{CV}$, sí que hay acuerdo en otorgar a ésta un marco multielemento, que tenga en cuenta qué es lo que las personas más valoran. El conjunto de todas esas dimensiones es lo que cabe considerar el constructo completo de la CV. 
La evaluación de la CV ha de ser realizada tanto desde la perspectiva subjetiva como desde la objetiva. Esto afecta a tres niveles, a saber: el microsistema, el mesosistema y el macrosistema (Schalock y Verdugo, 2002: 36-53). El microsistema engloba la parte subjetiva, a la cual se accede preguntando a la persona sobre la satisfacción de su vida en distintos ámbitos. El mesosistema hace alusión a la interacción de la persona con sus vecinos, la comunidad o la organización. Este aspecto indica la naturaleza objetiva de la CV. Por último, el macrosistema se relaciona con indicadores sociales vinculados a aspectos ambientales: la salud, el bienestar social, las amistades, niveles de vida, educación, seguridad pública, ocio, vecindario...

En resumen, a lo largo de los años el foco de análisis sobre la CV ha ido cambiando $\mathrm{y}$, actualmente, se centra en diversos ámbitos que hacen alusión a la persona. Aunque no tenemos definiciones claras sobre su significado, sí que disponemos de algunos marcadores y directrices que nos ayudan a evaluar la CV. Con ello, apreciamos que tiene un carácter multimodal y que ha de ser revisada tanto de manera objetiva como subjetiva, atendiendo a los niveles micro, meso y macro.

A continuación, aplicaremos este planteamiento al análisis de una enfermedad mental grave como es la esquizofrenia. Para ello nos basamos en el carácter multidimensional de la CV, en la necesidad de articular una perspectiva subjetiva y otra objetiva, y por último en esbozar algunos datos referentes a las dimensiones a las que se refieren Schalock y Verdugo.

\section{La calidad de vida en personas con esquizofrenia}

La medición de la CV de personas con esquizofrenia puede hacerse desde varias perspectivas: subjetiva (satisfacción de la vida por parte de la persona) u objetiva (actividades, participación en la sociedad y relaciones interpersonales) o incluso en ambas simultáneamente (3). Esta opción, ciertamente, resulta ser la más satisfactoria. Un planteamiento meramente objetivo deja fuera la percepción de la persona, quizá la más importante, porque, a fin de cuentas, es a quien hace referencia la CV. Un planteamiento subjetivo puede incurrir en 3 tipos de falacias: afectiva, cognitiva y de distorsión de la realidad (Katsching, 2000: 8-9). La falacia afectiva, que este autor considera la más importante, señala que una persona en un momento afectivo, como puede ser un estado depresivo, puede verbalizar sentimientos sobre su felicidad y CV de manera totalmente negativa, siendo incluso peores que los de un observador imparcial, o incluso que los del mismo paciente una vez recuperado. La falacia cognitiva es el resultado de una mala valoración por parte del paciente, debido a déficits intelectuales, como son los casos de demencias o retraso mental. La falacia de distorsión de la realidad se produce al realizar juicios sobre la CV basándose en delirios y/o alucinaciones.

Por tanto, creemos que la mejor manera de valorar la CV es una combinación de perspectivas subjetivas y objetivas. Por un lado, es necesario que la persona dé su opinión al respecto. Por otro, es importante observar qué aspectos son los que inciden y repercuten en la CV desde un punto de vista objetivo. Por esta razón, cuando evaluamos la CV han de reflejarse y distinguirse tres componentes, a saber: 1) bienestar/satisfacción subjetivos; 2) desempeño de roles (a nivel social); y 3) condiciones externas de vida, como pueden ser bienes materiales (propiedades, dinero...) y sociales (apoyo social, familiar, etc.) (Katschnig, 2000: 9-10). Esto indica la 
necesidad de articular los sistemas micro, meso y macro a los que hacen alusión Schalock R. y Verdugo M.

En efecto, es necesario un análisis conjunto de estos sistemas, y más si tenemos en cuenta que, en general, los pacientes con esquizofrenia tienen una CV más baja que la población general y los enfermos físicos (Alptkin et al., 2005; y Bobes y González, 2000: 162; Gómez, 2010: 43; y Sibitz, 2011). Pese a estos datos, se sabe que si hay una terapia combinada entre fármacos y psicoterapia puede haber una mejor CV. Se requiere una terapia biológica (medicación, etc.) y un análisis y valoración sobre cómo afecta a su vida, pero también un abordaje de los déficits psicosociales para tener una mejor satisfacción consigo mismo, así como aliviar los síntomas y signos de la enfermedad y mejorar las relaciones interpersonales, a fin de conseguir una mayor integración familiar y comunitaria. Por tanto, este modo de concebir la CV busca la comprensión bio-psico-social (Engel, 1977 y 1980) de la enfermedad, de su tratamiento y del modo en el que incide la patología en el día a día.

Los aspectos bio-psico-sociales son indicativos del grado de satisfacción o insatisfacción de la CV. Una baja CV en personas con esquizofrenia está asociada con los síntomas negativos, la depresión y los efectos extrapiramidales del tratamiento farmacológico (Narváez et al., 2008; Yamauchi et al., 2008). En cuanto al manejo terapéutico, el estudio de Hamann et al. (Hamann et al., 2005) demostró que la libre elección del tratamiento, el conocimiento de sus contraindicaciones y la importancia de su seguimiento, tienen como balance una mejor adherencia farmacológica y una reducción del número de recidivas. De modo que esto puede contribuir a un aumento de la CV al haber una mejor aceptación terapéutica y un mayor seguimiento farmacológico. En cuanto al tipo de fármaco, son elegidos los antipsicóticos atípicos, antes que los típicos, por tener una mayor contribución en el abordaje de los síntomas positivos y negativos, menos efectos adversos (principalmente extrapiramidales) y una estimulación de la función cognitiva (Gómez, 2010: 44-45), lo cual contribuye a un aumento de la CV. En particular, se ha demostrado que la olanzapina ayuda a la mejora de la CV a través del mejor control de los síntomas positivos y negativos, así como produciendo una mejora cognitiva y reduciendo de los síntomas depresivos (Wehmeier et al., 2007), aunque también la clozapina tiene efectos positivos en la cognición, principalmente en la fluidez verbal y la atención (Lee et al., 1999).

Otro tipo de repercusión de la esquizofrenia son los déficits asociados a las

Una baja CV en personas con esquizofrenia está asociada con los síntomas negativos, la depresión y los efectos extrapiramidales del tratamiento farmacológico. La libre elección del tratamiento, el conocimiento de sus contraindicaciones y la importancia de su seguimiento, tienen como balance una mejor el adherencia farmacológica y una reducción del número de recidivas. De modo que esto puede contribuir a un aumento de la CV al haber una mejor aceptación terapéutica y un mayor seguimiento farmacológico. funciones cognitivas (atención, percepción, memoria...), los cuales inciden negativamente en la $C V$, en particular en las dimensiones sociales. No hay que olvidar que estas personas pueden reflejar disfunción social, psicológica y/o ocupacional, lo cual se acentúa si hay una baja

fluidez verbal y problemas de atención. (Alptkin et al., 2004). Estos hallazgos son consistentes con el estudio de Ritsner (Ritsner, 2007), al encontrar también relación entre función ejecutiva, atención, memoria, habilidades motoras y CV. 
Al evaluar estos factores hemos de tener presente que la finalidad no es sólo la recuperación sintomatológica, sino también la reinserción psico-social. Para ello, es muy importante contar con una buena red social (amigos, familiares...). El apoyo interpersonal contribuye a una reducción del estigma social y aumenta el sentimiento de empowerment. Éste se refiere a que sea la propia persona la encargada de tomar decisiones por sí misma, al aumento de autoestima, optimismo, etc. $Y$ al contrario, una pobre red social aumenta la internalización del estigma y reduce el empowerment. La carencia de este soporte social favorece a la aparición de síntomas depresivos y esto se traduce en una reducción de la percepción subjetiva de la CV (Sibitz, 2011). Y es que los síntomas depresivos también tienen un impacto negativo en la vida cotidiana, al limitar la participación en las actividades diarias y en la relación con los amigos y familia (Narváez et al., 2008).

Una buena red social puede llevar también a una mejora en la CV relacionada con las actividades diarias. Las personas con esquizofrenia perciben como un factor importante para su vida las actividades relacionadas con el ocio, las tareas domésticas y las del cuidado personal. También dan gran importancia a los estudios y al trabajo. Y es que los que disponen de un trabajo y/o están realizando algún tipo de estudios tienen una mejor satisfacción y calidad de vida (Eklund, 2009). El trabajo posibilita que la persona estructure su tiempo, establezca vínculos interpersonales, disponga de ingresos económicos que le permiten tener más independencia, menor probabilidad de consumir algún tipo de droga, etc. (Bryson, Lysaker y Bell, 2002). Todos estos aspectos mejoran la CV.

Aunque estos aspectos psico-sociales mejora la CV de la persona, hay que valorar también qué tipo de CV tienen los familiares de estos pacientes. Esto es importante porque son ellos los encargados de su cuidado. La enfermedad mental impacta en la CV de los familiares, lo que a su vez repercute en el estado anímico de la persona con esquizofrenia. Por este motivo, se requiere una mayor interacción personal, un soporte emocional, una reducción del estrés en el ambiente familiar, una menor carga familiar, etc., para posibilitar una mayor CV para todos (Greenberg et al., 2006; Maldonado et al., 2012), pero también un abordaje psico-social del grupo familiar que le permita superar la carga física y emocional que supone el cuidado de una persona con esquizofrenia (Boyer et al., 2012). El objetivo de estas medidas también tiene influencia en el número de recidivas, al reducir el nivel Una buena red social puede llevar también a una mejora en la CV relacionada con las actividades diarias. Las personas con esquizofrenia perciben como un factor importante para su vida las actividades relacionadas con el ocio, las tareas domésticas y las del cuidado personal. También dan gran importancia a los estudios y al trabajo.

de ansiedad y estrés en el ambiente familiar. Una mayor satisfacción de la CV está asociada con una baja tasa de recaídas y, al contrario, una baja en la CV contribuye a un aumento en las recaídas (Boyer et al., 2013).

En definitiva, al evaluar la CV en pacientes con esquizofrenia hay que hacerlo tanto desde la perspectiva subjetiva como la objetiva. Para ello hay que valorar cuál es el grado de bienestar y satisfacción, el desempeño de roles sociales y las condiciones de vida materiales y sociales. 
y sus familias. Análisis bioético, conceptual y psicopatológico.

Y dado que queremos analizar qué satisfacción tiene la persona en los ámbitos bio-psico-sociales, hemos de realizar un análisis del tratamiento farmacológico y psicoterapéutico, para ver cómo incide la medicación y qué déficits psico-sociales tiene la persona que condiciona su vida cotidiana.

\section{Conclusiones}

Cuando las personas deciden cómo quieren enfocar su vida, tienen que hacerlo de manera responsable. Los proyectos de vida no son planes estéticos sino éticos. Al llevarlos a cabo hay que tener en cuenta unos mínimos cívicos que posibiliten una convivencia pacífica. Los intereses de los planes de vida han de ser críticos, justificados razonada y racionalmente. Por tanto, lo que se está reivindicando es que sea la propia persona quien decida cómo quiere llevar el proceso de vivir y de morir, es decir, qué entiende por $\mathrm{CV}$.

Pero apelar al derecho a una CV no basta para que se lleve a cabo. La definición de $\mathrm{CV}$ ha sido y es un gran problema conceptual, pues se carece de un acuerdo al respecto, lo cual dificulta su evaluación y aplicabilidad en los planes terapéuticos. Sin embargo, la bibliografía sí que comparte la idea de que ha de ser un constructo multidimensional, valorado tanto a nivel subjetivo como objetivo y que los resultados de su aplicabilidad han de ser analizados tanto a corto como a largo plazo. Además, su análisis ha de abarcar los niveles micro, meso y macro.

Al evaluar la CV de los pacientes con enfermedad mental grave, nos damos cuenta de la importancia de abordar esos tres niveles, pues las personas con esquizofrenia tienen una peor $\mathrm{CV}$ que el resto de la población. El enfoque terapéutico para tratar la enfermedad y aumentar así la CV ha de ser bio-psico-social, es decir, farmacológico y psicoterapéutico. Esto es importante, porque los síntomas negativos,

Las personas con esquizofrenia tienen una peor CV que el resto de la población. El enfoque terapéutico para tratar la enfermedad y aumentar así la $\mathrm{CV}$ ha de ser bio-psico-social, es decir, farmacológico y psicoterapéutico. Esto es importante, porque los síntomas negativos, los déficits cognitivos, la depresión y los efectos adversos del tratamiento farmacológico repercuten directamente en la CV de estas personas. los déficits cognitivos, la depresión y los efectos adversos del tratamiento farmacológico repercuten directamente en la CV de estas personas. Además, un buen abordaje de estos aspectos también reduce el nivel de recidivas. No hemos de olvidar que uno de los objetivos de los planes terapéuticos también ha de ser la reinserción psicosocial, dotando al enfermo de mayor protagonismo en la sociedad (empowerment). En cualquier caso, esta evaluación de la CV no ha de ceñirse exclusivamente a los pacientes, sino que se requiere también una evaluación de los familiares para ver qué nivel de satisfacción tienen, lo cual, a su vez, pude repercutir en la CV del paciente.

Este estudio sobre la valoración de la CV que hemos realizado, no ha tenido en cuenta ciertos aspectos o no los ha desarrollado suficientemente:

- $\quad$ No se ha querido dar ninguna definición de CV, sino que nos hemos basado en qué enfoques hay que trabajar para aumentar la calidad de vida. 
- Se han aceptado las dimensiones que proponen Schalock y Verdugo; sin embargo, no se ha examinado en detalle su contenido ni hemos analizado si de hecho son verdaderas dimensiones. Tampoco se han propuesto las de otros autores.

- Hemos dejado por desarrollar los instrumentos o modelos validados sobre la evaluación de la CV.

- Pese a la importancia de la psicoterapia en la mejora de la CV, tanto de los pacientes como de sus cuidadores, no se ha hecho referencia a ningún tipo de psicoterapia particular.

Por último, hay que insistir en la idea de que la CV ha de ser uno de los pilares fundamentales de los planes terapéuticos. La Psiquiatría no puede ceñirse únicamente a la erradicación de la sintomatología psicótica, sino que también ha de tener como referencia que las personas con enfermedades mentales tengan una mejor CV, para integrarlas en la sociedad y para que tengan herramientas con las que poder ser cada vez más autónomas. A fin de cuentas, estamos haciendo referencia a personas con una enfermedad mental y no simplemente a pacientes con esquizofrenia. Esta perspectiva también ha de completarse con la valoración de la CV de los familiares encargados del cuidado de la persona. La finalidad de los planes terapéuticos no ha de ser únicamente que la persona no tenga sintomatología psicótica, sino que también tenga una mejor CV. Esto no puede hacerse sin una estrategia terapéutica integral, bio-psico-social.

\section{Bibliografía}

- Kfksal, A.; YVldVz, A.; Berna, B.; Binnur, K.; KVvVrcVk, A.; Kemal, D.; Dogan, Y.; Ferdane, P.; Saida, Y. y Yahssin A. (2005). Is Quality of Life Associated with Cognitive Impairment in Schizophrenia? Progress in Neuro-Psychopharmacology \& Biological Psychiatry, 29, 239- 244.

- Awad, A., y Voruganti, L. (2012). Measuring Quality of Life in Patients with Schizophrenia. Pharmacoeconomics, 30(3), 183-195.

- Bobes J., y González G. Calidad de vida en la esquizofrenia (2000). En: Katschnig H., Freeman H., y Sartorious N. Calidad de vida en los trastornos mentales. Barcelona: Masson.

- Boyer, L.; Caqueo-Urízar, A.; Richieri, R.; Lancon, Ch.; Gutiérrez-Maldonado. K. y

- Auquier, P. (2012). Quality of Life among Caregivers of Patients with Schizophrenia: a Cross-Cultural Camparison of Chilean and French Families. Familiy Practice, 13, 42.

- Boyer, L.; Millier, A.; Perthame, E.; Aballea, S.; Auquier, P. y Toumi, M. (2013). Quality of life is predictive of relapse in Schizophrenia. BMC Psychiatry, 13, 15.

- Bryson, G.; Lysaker, P. y Bell, M. (2002). Quality of Life Benefits of Paid Work Activity in Schizophrenia. Schizophrenia Bulletin, 28, 2, 249-257.

- Camps, V. (2001). Una vida de calidad. Reflexiones sobre Bioética. Barcelona: Ares y Mares.

- Joyce, C.; Rosenheck, R.; Xu, W.; Thomas, J.; Henderson, W. y Dennis S. (2000). Quality of Life in Schizophrenia: A comparison of Instruments. Schizophrenia Bulletin, 26, 3, 659-666.

- Dworkin, R. (1993). Ética privada e igualitarismo político. Barcelona: Paidós. 
- Dworkin, R. (1998). El dominio de la vida. Una discusión acerca del aborto, la eutanasia y la libertad individual. Barcelona: Ariel.

- Eklund, M. (2009) Work Status, Daily Activities and Quality of Life among People with Severe Mental Illness. Qual Life Res, 18, 163-170.

- Engel, G. (1977). The Need for a New Medical Model: a Challenge for Biomedicine. Science, 196, 129-36.

- Engel, G. (1980). The Clinical Application of the Biopsychosocial Model. Am J Psychiatry, 137, 535-544.

- Gómez, M. (2010). Insight y calidad de vida objetiva y subjetiva en la esquizofrenia. Tesis Doctoral. Universidad de Barcelona. 2010. Disponible en: http://www.tdx.cat/TDX0407110-113006/. Visitada el 20 de diciembre de 2013.

- Greenberg, J.; Knudsen, K. y Aschbrenner, K. (2006). Prosocial Family Processes and the Quality of Life of Persons With Schizophrenia. Psychiatric Services, 57, 1771-1777.

- Hamann, J.; Cohen,R.; Leucht, S.; Busch, R. y Dipl.-Math. Kissling R., (2005). Do Patients with Schizophrenia Wish to Be Involved in Decisions About Their Medical Treatment? Am J Psychiatry, 162, 2382-2384.

- Hunt, S. (1997). The problem of Quality of Life. Quality of Life Research, 6, 205-212.

- Katschnig, H. (2000). "Utilidad del concepto de calidad de vida en los trastornos mentales". En: Katschnig, H.; Freeman, H. y Sartorious N. Calidad de vida en los trastornos mentales. Barcelona: Masson.

- Lee, M.; Jayathilake, K. y Meltzer, H. (1999). A Comparison of the Effect of Clozapine with Typical Neuroleptics on Cognitive Function in Neuroleptic-Responsive Schizophrenia. Schizophrenia Research, 37, 1-11.

- Lehman, A. (2000). "Instrumentos para medir la calidad de vida en los trastornos mentales". En: Katschnig, H.; Freeman H. y Sartorious N. Calidad de vida en los trastornos mentales. Barcelona: Masson.

- Maldonado, G.; Caqueo A.; Ferrer, M. y Fernández, P. (2012). Influencia de la percepción de apoyo y del funcionamiento social en la calidad de vida de pacientes con esquizofrenia y sus cuidadores. Psicothema, 24, 2, 255-262.

- Narváez J., Twamley E., Twamley, E.; McKibbi, C.; Heaton, R. y Patterson, T. (2008). Subjective and objective quality of life in schizophrenia. Schizophrenia Research, 98, 201-208.

- Nussbaum, M. (2007)- Las fronteras de la justicia. Consideraciones sobre la exclusión. Barcelona: Paidós.

- Ritsner, M. (2007). Predicting Quality of Life Impairment in Chronic Schizophrenia from Cognitive Variables. Qual Life Res, 16, 929-937.

- Shalock, R. y Verdugo, M. (2002) Calidad de vida. Manual para profesionales de la educación, salud y servicios sociales. Madrid: Alianza.

- Shalock, R. y Verdugo, M. (2009). "Revisión actualizada del concepto de calidad de vida". En: Verdugo, M. (dir.) Cómo mejorar la calidad de vida de las personas con discapacidad. Instrumentos y estrategias de evaluación. Salamanca: Amarú Ediciones.

- Sibitz, I.; Amering, M.; Unger, A.; Seyringer, M.; Bachmann, A.; Schrank, B.; Benesch, B.; Schulze, B. y Woppmann, A. (2011). The Impact of the Social Network, Stigma and Empowerment on the Quality of Life in Patients with Schizophrenia. European Psychiatry, 26, 28-33.

- Wehmeier, P.; Kluge, M.; Schneider, E.; Alexander, A.; Schacht, T;,

- Wagner, Th. y Schreiber, W. (2007). Quality of Life and Subjective Well-Being during Treatment with Antipsychotics in Out-patients with Schizophrenia. Progress in NeuroPsychopharmacology \& Biological Psychiatry, 31, 703-712

- WHOQOL Group. Development of the WHOQOL: Rationale and Current Status. Int J Mental Health. 1994;23:24-56.

- Yamauchi, K.; Hirofumi, A.; Yamauchi, K.; Aki, H.; Tomotake, Y.; Jun-Ichi, Iga; Numata, S.; Motoki, I.; Izaki, Y.; Tayoshi, S.; Kinouchi, S.; Sumitani, S.; Tayoshi, S.; Takikawa, Y.; Kaneda, Y.; Taniguchi, T.; Ishimoto, Y.; Ueno, S. y Ohmori, T. (2008). Predictors of 
y sus familias. Análisis bioético, conceptual y psicopatológico.

Subjective and Objective Quality of Life in Outpatients with Schizophrenia. Psychiatry and Clinical Neurosciences, 62, 404-411.

\section{Notas}

1.- Ejemplo de tales situaciones pueden ser el ingreso involuntario, el tratamiento ambulatorio involuntario, las medidas restrictivas (contención mecánica...), entre otras.

2.- Es posible sostener otra serie de dimensiones prototípicas, pero aquí sólo hacemos referencia a los trabajos de Schalock R. y Verdugo R. Para más información véase la lista que proponen Schalock y Verdugo (Schalock y Verdugo, 2002: 37) sobre varios autores y sus posiciones.

3.- Para un análisis sobre los distintos instrumentos sobre la evaluación de la CV en pacientes con esquizofrenia véase: Cramer et al., (Cramer et al., 2000), A. Lehman (Lehman, A., 2000), y Awad y Voruganti (Awad y Voruganti, 2012). 